Pacific

Journal of

Mathematics

\title{
CONTRACTING AN AXIALLY SYMMETRIC TORUS BY ITS HARMONIC MEAN CURVATURE
}

Christopher KIM 


\title{
CONTRACTING AN AXIALLY SYMMETRIC TORUS BY ITS HARMONIC MEAN CURVATURE
}

\author{
CHRISTOPHER KIM
}

\begin{abstract}
We consider the harmonic mean curvature flow of an axially symmetric torus whose axis is a closed geodesic, where the ambient space is a hyperbolic three-manifold. Assuming the initial surface is strictly convex and its harmonic mean curvature is less than $\frac{1}{2}$, we show that the evolving surface satisfies a curvature condition comparable to that of a perfectly symmetric torus evolving under harmonic mean curvature flow. In other words, we prove that $\lambda_{1} \approx e^{-t}, \lambda_{2} \approx e^{t}$ and $\lambda_{1} \lambda_{2} \approx 1$, where $\lambda_{1}$ and $\lambda_{2}$ are the principal curvatures of the evolving torus.
\end{abstract}

\section{Introduction}

We consider the contraction of a convex torus embedded in a hyperbolic 3-manifold to a closed geodesic using the harmonic mean of the principal curvatures. Each point on the torus whose axis is a closed geodesic moves in the normal direction pointing to its axis with a speed equal to the harmonic mean curvature. Let $\Sigma^{2}=$ $S^{1} \times S^{1}$ be a two-dimensional torus, $N^{3}$ a hyperbolic 3-manifold containing a closed geodesic and $\Phi_{0}: \Sigma^{2} \rightarrow N^{3}$ a smooth initial immersion of $\Sigma^{2}$ into $N^{3}$ centered at a closed geodesic. The evolution process is described by a one-parameter family of immersions $\Phi: \Sigma \times[0, T) \rightarrow N$ satisfying

(HMCF)

$$
\begin{aligned}
\frac{\partial \Phi(p, t)}{\partial t} & =-F(p, t) \cdot N(p, t), \\
\Phi(p, 0) & =\Phi_{0}(p) .
\end{aligned}
$$

Here, $F=\lambda_{1} \lambda_{2} /\left(\lambda_{1}+\lambda_{2}\right)$ is the harmonic mean curvature of $\Sigma_{t}:=\Phi(\Sigma, t)$ where $\lambda_{1}, \lambda_{2}$ are the principal curvatures and $N$ is the outward unit normal vector of $\Sigma_{t}$.

Andrews studied harmonic mean curvature flow (HMCF) of strictly convex compact hypersurfaces without boundary in Euclidean [Andrews 1994a] and Riemannian manifolds [1994b], showing that the evolving hypersurface converges to a round point in finite time. Other authors studied HMCF of hypersurfaces in Euclidean space under various curvature conditions [Caputo and Daskalopoulos

MSC2010: primary 53C44; secondary 35K55.

Keywords: harmonic mean curvature flow, hyperbolic manifold, closed geodesic. 
2009; Daskalopoulos and Hamilton 2006; Daskalopoulos and Sesum 2010; Dieter 2005] and showed that the evolving hypersurface converges, when it does, to a round point. In this paper, we are interested in surfaces converging to a closed geodesic, not a point, in hyperbolic 3-manifolds by HMCF. Examples of hypersurfaces in hyperbolic manifolds converging to a totally geodesic submanifold by HMCF were constructed in [Gulliver and Xu 2009]. However, only hypersurfaces at a constant distance from totally geodesic submanifolds were considered there, so the curvature flow problem reduced to analyzing simple ODEs. This paper generalizes parts of the results of Gulliver and $\mathrm{Xu}$ to axially symmetric surfaces. Recently in [Andrews et al. 2013], weakly convex hypersurfaces in Euclidean space containing cylindrical regions were shown to shrink to a line segment when the hypersurface is deformed by certain curvature function. However, curvatures of the evolving surface were not analyzed in that paper.

In this paper, we will obtain curvature estimates of an axially symmetric torus contracting to a closed geodesic in hyperbolic 3-manifold by HMCF. Analyzing the principal curvatures of a torus presents a novel problem since as the torus approaches the axis we expect the small principal curvature to converge zero and the large principal curvature to approach infinity. And the product of the principal curvatures is expected to be more or less constant since it equals 1 (see (1-1)) on a perfect torus whose axis is a closed geodesic. This kind of curvature estimate is different from the estimates obtained for spherical hypersurfaces in Theorem 4.1 of [Andrews 1994b] and Theorem 5.1 of [Huisken 1984], stating that the ratio of principal curvatures are uniformly bounded. We need to estimate each principal curvature separately to show that they exhibit contrasting dynamics but the product should remain bounded throughout the evolution process.

We will consider a torus $\Sigma^{2}$ embedded into a hyperbolic 3-manifold $N^{3}$ such that it is axially symmetric about a closed geodesic $\gamma: S^{1} \rightarrow N^{3}$. Let $r: S^{1} \rightarrow[0, R]$ be a generating function defined on $\gamma$. An axially symmetric torus can be constructed by revolving the graph of the generating function about the closed geodesic.

Theorem 1.1 (main theorem). Let $\Sigma_{0}$ be an axially symmetric torus around a closed geodesic $\gamma$ in a hyperbolic 3-manifold $N$, generated by revolving a graph of $r: S^{1} \rightarrow \mathbb{R}^{+}$about $\gamma$. Assume $\Sigma_{0}$ is strictly convex and $\max _{x \in \Sigma_{0}} F(x)<\frac{1}{2}$ where $F(x)$ is the harmonic mean curvature at $x \in \Sigma_{0}$. Then, the solution of the $H M C F$ with initial surface $\Sigma_{0}$ exists for all $t \in[0, \infty)$ and remains strictly convex. The evolving surface converges to the closed geodesic exponentially fast and the principal curvatures satisfy $\lambda_{1} \approx e^{-t}, \lambda_{2} \approx e^{t}$ and $\lambda_{1} \lambda_{2} \approx 1$.

Notation. Uniform constants are denoted by $C_{i}$. The same symbol $C$ might imply different constants from line to line. The approximation symbol $f \approx g$ denotes that there exist $C_{1}, C_{2}>0$ such that $C_{1} g \leq f \leq C_{2} g$. 
Remarks. (1) The reason we impose the curvature condition $\max _{\Sigma_{0}} F<\frac{1}{2}$ is that for a perfectly symmetric torus we have $0<F(r)<\frac{1}{2}$ for all $r \in(0, \infty)$; thus perfectly symmetric tori of any radius satisfy the condition. This can be easily seen as follows: Since the principal curvatures of a perfect torus are $\lambda_{1}=\tanh r$ and $\lambda_{2}=\operatorname{coth} r$ (by the Riccati equation, $\lambda_{i}^{\prime}+\lambda_{i}^{2}=1$ ), the harmonic mean curvature is

$$
F=\frac{1}{(\operatorname{coth} r)^{-1}+(\tanh r)^{-1}}=\frac{1}{\operatorname{coth} r+\tanh r} .
$$

Thus,

$$
\frac{d F}{d r}=\frac{1}{\sinh ^{2} r+\cosh ^{2} r}>0 \text { for all } r
$$

But

$$
\lim _{r \rightarrow 0} \operatorname{coth} r=\infty, \quad \lim _{r \rightarrow 0} \tanh r=0, \quad \lim _{r \rightarrow \infty} \operatorname{coth} r=\lim _{r \rightarrow \infty} \tanh r=1 .
$$

Therefore

$$
\lim _{r \rightarrow 0} F=0, \quad \lim _{r \rightarrow \infty} F=\frac{1}{2}, \quad 0<F(r)<\frac{1}{2} .
$$

(2) The HMCF of a perfectly symmetric torus whose axis is a closed geodesic in a hyperbolic manifold was considered in Theorem 3 of [Gulliver and $\mathrm{Xu}$ 2009]. The authors showed that the radius $r(t)$ of the evolving torus satisfies

$$
r(t)=\frac{1}{2} \sinh ^{-1}\left(e^{-t} \sinh 2 r_{0}\right) \approx e^{-t},
$$

where $r_{0}$ is the radius of the initial torus. Since the principal curvatures of perfect torus are $\lambda_{1}=\tanh r$ and $\lambda_{2}=\operatorname{coth} r$, we obtain the asymptotic estimates of both principal curvatures:

$$
\lambda_{1} \approx e^{-t}, \quad \lambda_{2} \approx e^{t}, \quad \lambda_{1} \lambda_{2}=1 .
$$

The main theorem of this paper shows that the principal curvatures of an axially symmetric torus contracting to a closed geodesic under HMCF retain the curvature estimates (1-1) of an evolving perfectly symmetric torus.

The paper is organized as follows. In Section 2, we derive essential geometric quantities available on axially symmetric spaces. In Section 3, we prove the short and long time existence of HMCF of axially symmetric torus and discuss the preservation of convexity of the surface. We derive the evolution equations of important geometric quantities in Section 4. In Section 5, we prove that the evolving surface remains a graph throughout the deformation process and also prove that $\lambda_{2} \approx e^{t}$. Along the way, we obtain the optimal estimate $\lambda_{1} \approx e^{-t}$ and conclude that $\lambda_{1} \lambda_{2} \approx 1$. 


\section{Axially symmetric spaces}

In this section, we will use the orthonormal frames to derive geometric quantities defined on axially symmetric surfaces. A similar computation was carried out in [Cabezas-Rivas and Miquel 2009] for general rotationally symmetric spaces. In the neighborhood of the closed geodesic, the hyperbolic metric can be expressed in Fermi coordinates as $d s^{2}=d r^{2}+h(r)^{2} d \theta^{2}+b(r)^{2} d z^{2}$ where $r$ is the distance from the axis, $\theta$ is the angular unit of the circle perpendicular to the axis, $z$ is the position along the axis and $b(r)=\cosh r, h(r)=\sinh r$. We have the following orthonormal frames in $(n+1)$-dimensional rotationally symmetric space.

$$
E_{0}:=E_{r}=\frac{\partial}{\partial r}, \quad E_{1}:=E_{z}=\frac{1}{b(r)} \frac{\partial}{\partial z}, \quad E_{i}=\frac{1}{h(r)} e_{i} \quad \text { for } i=2, \ldots, n,
$$

where $e_{i}$ is an orthonormal frame of $S^{n-1}$ with the standard metric. Its dual orthonormal coframe is given by

$$
\theta^{r}=d r, \quad \theta^{z}=b(r) d z, \quad \theta^{i}=h(r) e_{i} \quad \text { for } i=2, \ldots, n .
$$

In these frames, the Cartan connection form $\omega_{a}^{b}$ defined by $d \theta^{b}=-\sum_{a=0}^{n} \omega_{a}^{b} \wedge \theta^{a}$ is given by

$$
\omega_{r}^{z}=\frac{b^{\prime}(r)}{b(r)} \theta^{z}, \quad \omega_{r}^{i}=\frac{h^{\prime}(r)}{h(r)} \theta^{i}, \quad \omega_{z}^{i}=0, \quad \omega_{j}^{i}={ }^{S} \omega_{j}^{i}
$$

where ${ }^{S} \omega_{j}^{i}$ represents the Cartan connection form on $S^{n-1}$. The covariant derivatives of the orthonormal frames can be computed from the equation $\bar{\nabla}_{X} E_{a}=$ $\sum_{b=0}^{n} \omega_{a}^{b}(X) E_{b}$ and their results are given below. We denote the covariant derivative defined on the ambient manifold by $\bar{\nabla}$ and the covariant derivative on the hypersurface by $\nabla$. The symbol ' denotes the derivative with respect to $r$ and subscripts of $r$ mean the derivative with respect to $z$. For $i=2, \ldots, n$,

$$
\bar{\nabla}_{E_{r}} E_{r}=0, \quad \bar{\nabla}_{E_{z}} E_{r}=\frac{b^{\prime}(r)}{b(r)} E_{z}, \quad \bar{\nabla}_{E_{i}} E_{r}=\frac{h^{\prime}(r)}{h(r)} E_{i}
$$$$
\bar{\nabla}_{E_{r}} E_{z}=0, \quad \bar{\nabla}_{E_{z}} E_{z}=-\frac{b^{\prime}(r)}{b(r)} E_{r}, \quad \bar{\nabla}_{E_{i}} E_{z}=0,
$$

$$
\bar{\nabla}_{E_{r}} E_{i}=0, \quad \bar{\nabla}_{E_{z}} E_{i}=0, \quad \quad \bar{\nabla}_{E_{i}} E_{j}=-\frac{h^{\prime}(r)}{h(r)} \delta_{i j} E_{r}+{ }^{S} \omega_{j}^{k}\left(E_{i}\right) E_{k} .
$$

For a hypersurface constructed by revolving the graph of a generating function $r: S^{1} \rightarrow \mathbb{R}^{+}$, the tangent vector $\sigma$ of the generating curve and the unit normal vector $N$ of the hypersurface are given by

$$
\sigma=\frac{1}{\sqrt{r_{z}^{2}+b^{2}}}\left(r_{z} E_{r}+b E_{z}\right), \quad N=\frac{1}{\sqrt{r_{z}^{2}+b^{2}}}\left(b E_{r}-r_{z} E_{z}\right) .
$$


The principal curvatures of the hypersurface in the direction of $\sigma$ and $E_{i}$ are

$$
\begin{aligned}
& \lambda_{1}=\left\langle\bar{\nabla}_{\sigma} \sigma, N\right\rangle=\frac{1}{\sqrt{r_{z}^{2}+b^{2}}}\left(\frac{-r_{z z} b+r_{z}^{2} b^{\prime}}{r_{z}^{2}+b^{2}}+b^{\prime}\right), \\
& \lambda_{i}=\left\langle\bar{\nabla}_{E_{i}} E_{i}, N\right\rangle=\frac{b}{\sqrt{r_{z}^{2}+b^{2}}} \frac{h^{\prime}}{h}=u \frac{h^{\prime}}{h} \quad \text { for } i=2, \ldots, n,
\end{aligned}
$$

respectively. Note the hypersurfaces of revolution is generated by a graph if

$$
u:=\left\langle E_{r}, N\right\rangle=\frac{b}{\sqrt{r_{z}^{2}+b^{2}}}
$$

is greater than 0 ; equivalently, $v:=u^{-1}$ is finite. Note that $u \leq 1$ by its definition.

\section{Short and long time existence and preserving convexity}

In this section, we first prove the short time existence of HMCF of axially symmetric torus and review the long time existence and preservation of convexity proved in [Gulliver and Xu 2009]. Let $W_{i}^{j}=h_{i k} g^{k j}$ be the Weingarten map of $\Sigma_{t}$, where $h_{i j}$ is the second fundamental form and $g_{i j}$ is the induced metric on $\Sigma_{t}$. We can view the harmonic mean curvature function as $F\left(W_{i}^{j}\right)=f\left(\lambda\left(W_{i}^{j}\right)\right)$, where $\lambda\left(W_{i}^{j}\right)=\left(\lambda_{1}, \lambda_{2}\right)$ is the set of eigenvalues of $W_{i}^{j}$ and $f\left(\lambda_{1}, \lambda_{2}\right)=\lambda_{1} \lambda_{2} /\left(\lambda_{1}+\lambda_{2}\right)$. Let us first discuss the short time existence of HMCF when the flow equation is cast in terms of the graph function. If we express (HMCF) in terms of the graph function using

$$
\left\langle\frac{\partial \phi}{\partial t}, N\right\rangle=F
$$

we obtain

$$
\text { (3-1) } \frac{\partial r}{\partial t}=-\frac{r_{z z}-2 \tanh (r) r_{z}^{2}-\sinh r \cosh r}{\tanh (r) r_{z z}-\left(2 \tanh ^{2} r+1\right) r_{z}^{2}-\sinh ^{2} r-\cosh ^{2} r}, \quad r(z, 0)=r_{0}(z)
$$

for all $(z, t) \in S^{1} \times[0, T)$. Since the initial surface is assumed to be strictly convex, from (2-3) and (2-4) we find that at $t=0$

$$
\tilde{\lambda}_{1}:=-r_{z z}+2 \tanh (r) r_{z}^{2}+\sinh r \cosh r>0 .
$$

We consider positive solutions

$$
r>0 .
$$

We define $C^{\alpha}\left(S^{1}\right)$ to be the set of standard Hölder continuous functions on $S^{1}$ and $C^{2+\alpha}\left(S^{1}\right)$ to be a space of functions $g$ on $S^{1}$ such that $g, g_{z}, g_{z z} \in C^{\alpha}\left(S^{1}\right)$. We set $Q_{\tau}=S^{1} \times[0, \tau]$ for some $\tau>0$ and define $C^{2+\alpha}\left(Q_{\tau}\right)$ to be a space of functions $g$ on $Q_{\tau}$ such that $g_{t}, g, g_{z}, g_{z z} \in C^{\alpha}\left(S^{1}\right)$. 
Lemma 3.1. Let $r_{0} \in C^{2+\alpha}\left(S^{1}\right)$. There exists some $t_{0}>0$ such that a unique solution $r \in C^{2+\alpha}\left(S^{1} \times\left[0, t_{0}\right]\right)$ solves (3-1).

Proof. Let $M: C^{2+\alpha}\left(Q_{\tau}\right) \rightarrow C^{\alpha}\left(Q_{\tau}\right)$ be a fully nonlinear operator defined by

$$
M(r)=r_{t}-F\left(z, t, r, r_{z}, r_{z z}\right),
$$

where

$$
F\left(z, t, r, r_{z}, r_{z z}\right)=-\frac{r_{z z}-2 \tanh r r_{z}^{2}-\sinh r \cosh r}{\tanh r r_{z z}-\left(2 \tanh ^{2} r+1\right) r_{z}^{2}-\sinh ^{2} r-\cosh ^{2} r} .
$$

Consider the linearization of $M$ around a function $r \in C^{2+\alpha}\left(Q_{\tau}\right)$ such that $\left\|r-r_{0}\right\|<\delta$ for some $\delta>0$. If we choose $\delta$ small enough, any such $r$ will satisfy conditions (3-2) and (3-3) since the initial condition $r_{0} \in C^{2+\alpha}\left(S^{1}\right)$ satisfies those conditions. Then, the linearized equation around the function $r$, namely

$$
\frac{\partial \tilde{r}}{\partial t}=D F(r)(\tilde{r})=\alpha\left(r, r_{z}, r_{z z}\right) \tilde{r}_{z z}+\beta\left(r, r_{z}, r_{z z}\right) \tilde{r}_{z}+\gamma\left(r, r_{z}, r_{z z}\right) \tilde{r},
$$

where

$$
\begin{aligned}
\alpha= & \frac{-r_{z}^{2}-\cosh ^{2} r}{\left(\tanh r \tilde{\lambda}_{1}+r_{z}^{2}+\cosh ^{2} r\right)^{2}}, \\
\beta= & \frac{\left(4 \tanh ^{2} r-4 \tanh ^{2} r-2\right) r_{z} \tilde{\lambda}_{1}+4 \tanh r r_{z}\left(r_{z}^{2}+\cosh ^{2} r\right)}{\left(\tanh r \tilde{\lambda}_{1}+r_{z}^{2}+\cosh ^{2} r\right)^{2}}, \\
\gamma= & {\left[\left(\frac{r_{z z}}{\cosh ^{2} r}-\frac{2 \tanh r}{\cosh ^{2} r} r_{z}^{2}-3 \sinh r \cosh r+\sinh ^{2} r \tanh r\right) \tilde{\lambda}_{1}\right.} \\
& \left.+\left(\frac{2 r_{z}^{2}}{\cosh ^{2} r}+\cosh ^{2} r+\sinh ^{2} r\right)\left(r_{z}^{2}+\cosh ^{2} r\right)\right] /\left(\tanh r \tilde{\lambda}_{1}+r_{z}^{2}+\cosh ^{2} r\right)^{2}
\end{aligned}
$$

satisfy

$$
\inf _{Q_{\tau}} \alpha\left(r, r_{z}, r_{z z}\right)>\mu>0 \text { for some } \mu \text { and } \alpha, \beta, \gamma \in C^{\alpha}\left(Q_{\tau}\right) \text {. }
$$

By standard theory for linear parabolic PDEs, the linearized equation (3-4) with the initial condition $\tilde{r}_{0} \in C^{2+\alpha}\left(S^{1}\right)$ has a unique solution $\tilde{r} \in C^{2+\alpha}\left(Q_{\tau}\right)$. Applying the inverse function theorem for Banach spaces (see [Daskalopoulos and Hamilton 1999, Theorem 8.5]), we conclude that there exists $t_{0}>0$ such that (3-1) has a unique solution $r \in C^{2+\alpha}\left(Q_{t_{0}}\right)$.

Remark. The fully nonlinear equation (3-1) is, in fact, uniformly parabolic due to $C^{1}$ and $C^{2}$ estimates of $r$ (Corollary 5.7).

In [Gulliver and $\mathrm{Xu}$ 2009, Theorem 6], it is proved that the solution of (HMCF) exists for infinite time and the evolving surface remains strictly convex. We will restate the theorem dividing it into two parts: the first stating the lower bound of 
the harmonic mean curvature (HMC) and the second stating its upper bound. We will give the entire proof of the second part since some estimates used in the proof will be improved in Section 4 in order to obtain the asymptotically optimal upper bound for HMC.

Theorem 3.2 [Gulliver and Xu 2009, Theorem 6]. Let $N^{3}$ be a hyperbolic manifold. If the initial surface is strictly convex, then $F(x, t) \geq\left(\min _{M_{0}} F\right) e^{-t}$ as long as the solution of HMCF exists. In other words, the surface remains strictly convex.

Theorem 3.3. Let $N^{3}$ be a hyperbolic manifold. Assume that the initial hypersurface is strictly convex and $\max _{\Sigma_{0}} F<\frac{1}{2}$. Then, the solution of HMCF exists for infinite time and $\max _{\Sigma_{t}} F \leq C e^{-t / 2}$ for some constant $C$ and for all $t \in[0, \infty)$.

Remark. Note that $f \leq \lambda_{1} \leq 2 f$ if $\lambda_{i}>0$. Therefore, the theorem implies that $\max _{\Sigma_{t}} \lambda_{1} \leq C e^{-t / 2}$, where $\lambda_{1}$ is the smallest principal curvature. Together with Theorem 3.2, we obtain $C_{1} e^{-t} \leq F \leq C_{2} e^{-t / 2}$.

Proof. We find the upper bound for $F$ by analyzing the evolution equation of $F$. We set

$$
\mathscr{L}=\frac{\partial F}{\partial h_{i}^{j}} \nabla_{i} \nabla^{j},
$$

which is an elliptic operator as long as the hypersurface is strictly convex.

$$
\begin{aligned}
\frac{\partial F}{\partial t} & =\mathscr{L}(F)+F\left\langle\dot{F}, W^{2}\right\rangle+F\left\langle\dot{F}^{i j}, R_{i 0 j 0}\right\rangle \\
& =\mathscr{L}(F)+\sum_{i} F \frac{\partial f}{\partial \lambda_{i}}\left(\lambda_{i}^{2}+R_{i 0 i 0}\right) \\
& \leq \mathscr{L}(F)+\sum_{i} F^{3}-\sum_{i} F \frac{\partial f}{\partial \lambda_{i}} \\
& =\mathscr{L}(F)+2 F^{3}-F^{3} \sum_{i} \lambda_{i}^{-2} \leq \mathscr{L}(F)+2 F^{3}-\frac{1}{2} F .
\end{aligned}
$$

By the maximum principle, we can solve the following ODE and obtain an upper bound for $F(x, t)$ :

$$
\frac{d \tilde{F}}{d t}=2 \tilde{F}^{3}-\frac{1}{2} \tilde{F}, \quad \tilde{F}(0)=\max _{x \in M} F(x, 0) .
$$

The solution of the ODE is $\tilde{F}(t)^{-2}=\left(\tilde{F}(0)^{-2}-4\right) e^{t}+4$, so we have $F(x, t) \leq \tilde{F}(t)$ for all $x \in M$ as long as the solution of HMCF exists. For the proof of infinite time existence, see [Gulliver and Xu 2009, Theorem 6].

Since disjoint surfaces remain disjoint under HMCF by the maximum principle, given a torus whose axis is a closed geodesic, two perfect tori enclosing it from inside and outside, which are called barriers, will remain disjoint throughout the flow; thus, the radius of the evolving torus is comparable to the radii of the barriers. 
Lemma 3.4. Let $r$ be the generating function of an axially symmetric torus evolving by $H M C F$. Then there exist $C_{1}$ and $C_{2}$ such that $C_{1} e^{-t} \leq r(x, t) \leq C_{2} e^{-t}$ for all $x \in \Sigma_{t}$ as long as the solution of HMCF exists.

\section{Evolution equations}

To show that the surface of revolution remains a graph over the closed geodesic, it is sufficient to prove that $v$ remains uniformly bounded for all time. To this end, we first derive the evolution equations of $r$ (Lemma 4.1) and $v$ (Lemma 4.3). From now on we will only consider the case $n=2$.

Lemma 4.1. The generating function satisfies following evolution equation.

$$
\left(\frac{\partial}{\partial t}-\mathscr{L}\right) r=\left(\lambda_{1} \frac{\partial f}{\partial \lambda_{1}}-f\right) u-\frac{\partial f}{\partial \lambda_{1}} \frac{b^{\prime}}{b} u^{2}-\frac{\partial f}{\partial \lambda_{2}} \frac{h^{\prime}}{h}\left(1-u^{2}\right) .
$$

Proof. Let us compute $\frac{\partial r}{\partial t}$ and $\mathscr{L}$.

$$
\frac{\partial r}{\partial t}=\left\langle\frac{\partial}{\partial r}, \frac{\partial X}{\partial t}\right\rangle=-f u .
$$

We choose a geodesic coordinate $\partial_{1}=\sigma, \partial_{2}=E_{2}$ at a fixed point such that $g_{i j}=\delta_{i j}$ and $h_{i j}=\lambda_{i} \delta_{i j}$ for $i, j=1,2$. Since $\nabla_{\sigma} \sigma=0$ and $E_{2}(r)=0$,

$$
\mathscr{L} r=\dot{F}^{k l} \nabla_{k} \nabla^{l} r=\frac{\partial f}{\partial \lambda_{1}} \nabla_{\sigma} \nabla_{\sigma} r+\frac{\partial f}{\partial \lambda_{2}} \nabla_{E_{2}} \nabla_{E_{2}} r=\frac{\partial f}{\partial \lambda_{1}} \sigma \sigma(r)-\frac{\partial f}{\partial \lambda_{2}}\left(\nabla_{E_{2}} E_{2}\right) r .
$$

Let us first compute the term $\sigma \sigma(r)$. By (2-1)-(2-5),

and

$$
\sigma(r)=\left\langle\sigma, \frac{\partial}{\partial r}\right\rangle=\frac{r_{z}}{\sqrt{r_{z}^{2}+b^{2}}}=-\left\langle N, E_{z}\right\rangle
$$

$$
\begin{aligned}
\sigma \sigma(r) & =-\sigma\left\langle N, E_{z}\right\rangle=-\left\langle\bar{\nabla}_{\sigma} N, E_{z}\right\rangle-\left\langle N, \bar{\nabla}_{\sigma} E_{z}\right\rangle \\
& =-\left\langle\lambda_{1} \sigma, E_{z}\right\rangle-\left\langle N,-\frac{b^{\prime}}{\sqrt{r_{z}^{2}+b^{2}}} E_{r}\right\rangle=-\lambda_{1} u+\frac{b^{\prime}}{b} u^{2} .
\end{aligned}
$$

On the other hand,

$$
-\left(\nabla_{E_{2}} E_{2}\right) r=-\left\langle\bar{\nabla}_{E_{2}} E_{2}, \sigma\right\rangle \sigma(r)=\frac{h^{\prime}}{h}\left(1-u^{2}\right) .
$$

Combining (4-1) and (4-2), we obtain

$$
\mathscr{L} r=\frac{\partial f}{\partial \lambda_{1}}\left(-\lambda_{1} u+\frac{b^{\prime}}{b} u^{2}\right)+\frac{\partial f}{\partial \lambda_{2}} \frac{h^{\prime}}{h}\left(1-u^{2}\right),
$$

and this finishes the proof of the lemma.

It is straightforward to derive the evolution equation of $\phi(r)$ for a smooth function $\phi: \mathbb{R} \rightarrow \mathbb{R}$. 
Lemma 4.2. The following evolution equation is satisfied by $\phi \circ r: \Sigma \times[0, \infty) \rightarrow \mathbb{R}$ : $\left(\frac{\partial}{\partial t}-\mathscr{L}\right) \phi(r)=\phi^{\prime}\left[\left(\lambda_{1} \frac{\partial f}{\partial \lambda_{1}}-f\right) u-\frac{\partial f}{\partial \lambda_{1}} \frac{b^{\prime}}{b} u^{2}-\frac{\partial f}{\partial \lambda_{2}} \frac{h^{\prime}}{h}\left(1-u^{2}\right)\right]-\phi^{\prime \prime} \frac{\partial f}{\partial \lambda_{1}}\left(1-u^{2}\right)$.

Proof. We compute

$$
\phi^{\prime \prime} \dot{F}^{k l} \nabla_{k} r \nabla_{l} r=\phi^{\prime \prime} \frac{\partial f}{\partial \lambda_{1}}(\sigma(r))^{2}=\phi^{\prime \prime} \frac{\partial f}{\partial \lambda_{1}}\left\langle E_{z}, N\right\rangle^{2}=\phi^{\prime \prime} \frac{\partial f}{\partial \lambda_{1}}\left(1-u^{2}\right) .
$$

Lemma 4.3. The gradient function $v=u^{-1}$ satisfies the evolution equation

$$
\begin{aligned}
\left(\frac{\partial}{\partial t}-\mathscr{L}\right) v=-\frac{2}{v} \dot{f}^{k l} \nabla_{k} v \nabla_{l} v-\frac{\partial f}{\partial \lambda_{1}} & \left(\frac{b^{\prime}}{b}\right)^{\prime}\left(v-v^{-1}\right)-\frac{\partial f}{\partial \lambda_{1}} v\left(v^{-1} \frac{b^{\prime}}{b}-\lambda_{1}\right)^{2} \\
& +\left(\frac{\partial f}{\partial \lambda_{2}} \frac{b^{\prime} h^{\prime}}{b h}-\frac{\partial f}{\partial \lambda_{2}}\left(\frac{h^{\prime}}{h}\right)^{\prime}+\frac{\partial f}{\partial \lambda_{1}}\left(\frac{b^{\prime}}{b}\right)^{2}\right) v \\
& -\frac{\partial f}{\partial \lambda_{2}} \lambda_{2} \frac{b^{\prime}}{b}+\left(\frac{\partial f}{\partial \lambda_{2}}\left(\frac{h^{\prime}}{h}\right)^{\prime}-\frac{\partial f}{\partial \lambda_{1}}\left(\frac{b^{\prime}}{b}\right)^{2}\right) v^{-1} .
\end{aligned}
$$

Proof. Let us first compute $\dot{F}^{k l} \nabla_{k} \nabla^{l} u$ by choosing the geodesic coordinate at a fixed point as before:

$$
\dot{F}^{k l} \nabla_{k} \nabla_{l} u=\frac{\partial f}{\partial \lambda_{1}} \sigma \sigma(u)-\frac{\partial f}{\partial \lambda_{2}}\left(\nabla_{E_{2}} E_{2}\right) u .
$$

From (2-1) and (2-2), we get $\bar{\nabla}_{\sigma} E_{r}=u \frac{b^{\prime}}{b} E_{z}$. Substituting, we obtain

$$
\sigma(u)=\sigma\left\langle E_{r}, N\right\rangle=\left\langle\bar{\nabla}_{\sigma} E_{r}, N\right\rangle+\left\langle E_{r}, \bar{\nabla}_{\sigma} N\right\rangle=\left(u \frac{b^{\prime}}{b}-\lambda_{1}\right)\left\langle E_{z}, N\right\rangle .
$$

As preparation for calculating $\sigma \sigma(u)$, we first observe that, by (2-2) and (4-3),

$$
\sigma\left(u \frac{b^{\prime}}{b}\right)=\left[\left(u \frac{b^{\prime}}{b}-\lambda_{1}\right) \frac{b^{\prime}}{b}-u\left(\frac{b^{\prime}}{b}\right)^{\prime}\right]\left\langle E_{z}, N\right\rangle .
$$

From (2-1) and (2-2), we see $\bar{\nabla}_{\sigma} E_{z}=-u\left(b^{\prime} / b\right) E_{r}$, and get

$$
\sigma\left\langle E_{z}, N\right\rangle=\left\langle-u \frac{b^{\prime}}{b} E_{r}, N\right\rangle+\left\langle E_{z}, \lambda_{1} \sigma\right\rangle=-u\left(u \frac{b^{\prime}}{b}-\lambda_{1}\right) .
$$

Then,

$$
\sigma \sigma(u)=\left[\left(u \frac{b^{\prime}}{b}-\lambda_{1}\right) \frac{b^{\prime}}{b}-u\left(\frac{b^{\prime}}{b}\right)^{\prime}\right]\left(1-u^{2}\right)-\sigma\left(\lambda_{1}\right)\left\langle E_{z}, N\right\rangle-u\left(u \frac{b^{\prime}}{b}-\lambda_{1}\right)^{2},
$$

where we used that $\left\langle E_{z}, N\right\rangle^{2}=1-u^{2}$. By (2-1) and (4-3), it is straightforward to compute

$$
\left(\nabla_{E_{2}} E_{2}\right) u=\left\langle\bar{\nabla}_{E_{2}} E_{2}, \sigma\right\rangle \sigma(u)=\frac{h^{\prime}}{h}\left(u \frac{b^{\prime}}{b}-\lambda_{1}\right)\left(1-u^{2}\right) .
$$

We finally obtain 


$$
\begin{aligned}
\dot{F}^{k l} \nabla_{k} \nabla_{l} u=\frac{\partial f}{\partial \lambda_{1}}( & {\left[\left(u \frac{b^{\prime}}{b}-\lambda_{1}\right) \frac{b^{\prime}}{b}-u\left(\frac{b^{\prime}}{b}\right)^{\prime}\right]\left(1-u^{2}\right) } \\
& \left.\quad-\sigma\left(\lambda_{1}\right)\left\langle E_{z}, N\right\rangle-u\left(u \frac{b^{\prime}}{b}-\lambda_{1}\right)^{2}\right) \\
& -\frac{\partial f}{\partial \lambda_{2}} \frac{h^{\prime}}{h}\left(u \frac{b^{\prime}}{b}-\lambda_{1}\right)\left(1-u^{2}\right) .
\end{aligned}
$$

In order to compute $\partial u / \partial t$, we will use the identities

$$
\frac{\partial N}{\partial t}=\nabla F, \quad \frac{\partial E_{r}}{\partial t}=-F \bar{\nabla}_{N} E_{r}=-F\left\langle E_{z}, N\right\rangle \frac{b^{\prime}}{b} E_{z} .
$$

Then,

$$
\begin{aligned}
\frac{\partial u}{\partial t}= & \frac{\partial}{\partial t}\left\langle N, E_{r}\right\rangle=\sigma(F)\left\langle\sigma, E_{r}\right\rangle-F\left(1-u^{2}\right) \frac{b^{\prime}}{b} \\
= & -\frac{\partial f}{\partial \lambda_{1}} \sigma\left(\lambda_{1}\right)\left\langle E_{z}, N\right\rangle-\frac{\partial f}{\partial \lambda_{2}} \sigma\left(\lambda_{2}\right)\left\langle E_{z}, N\right\rangle-F\left(1-u^{2}\right) \frac{b^{\prime}}{b} \\
=-\frac{\partial f}{\partial \lambda_{1}} \sigma\left(\lambda_{1}\right)\left\langle E_{z}, N\right\rangle-\frac{\partial f}{\partial \lambda_{2}}\left[\left(u \frac{b^{\prime}}{b}-\lambda_{1}\right) \frac{h^{\prime}}{h}-u\left(\frac{h^{\prime}}{h}\right)^{\prime}\right]\left(1-u^{2}\right) & -F\left(1-u^{2}\right) \frac{b^{\prime}}{b},
\end{aligned}
$$

where we used $\left\langle\sigma, E_{r}\right\rangle=-\left\langle E_{z}, N\right\rangle$ in the third equation and in the last equation we substituted

$$
\sigma\left(\lambda_{2}\right)=\sigma\left(u \frac{h^{\prime}}{h}\right)=\left[\left(u \frac{b^{\prime}}{b}-\lambda_{1}\right) \frac{h^{\prime}}{h}-u\left(\frac{h^{\prime}}{h}\right)^{\prime}\right]\left\langle E_{z}, N\right\rangle .
$$

From (4-4) and (4-5), we derive

$$
\begin{aligned}
\left(\frac{\partial}{\partial t}-\dot{F}^{k l} \nabla_{k} \nabla_{l}\right) u & =\frac{\partial f}{\partial \lambda_{2}} u\left(1-u^{2}\right)\left(\frac{h^{\prime}}{h}\right)^{\prime}-F\left(1-u^{2}\right) \frac{b^{\prime}}{b} \\
& -\frac{\partial f}{\partial \lambda_{1}}\left[\left(u \frac{b^{\prime}}{b}-\lambda_{1}\right) \frac{b^{\prime}}{b}-u\left(\frac{b^{\prime}}{b}\right)^{\prime}\right]\left(1-u^{2}\right)+\frac{\partial f}{\partial \lambda_{1}} u\left(u \frac{b^{\prime}}{b}-\lambda_{1}\right)^{2} .
\end{aligned}
$$

By the definition of $v=u^{-1}$, we have $\dot{F}^{k l} \nabla_{k} \nabla_{l} u=-\frac{1}{v^{2}} \dot{F}^{k l} \nabla_{k} \nabla_{l} v+\frac{2}{v^{3}} \dot{F}^{k l} \nabla_{k} v \nabla_{l} v$ and $\partial u / \partial t=-\left(1 / v^{2}\right) \partial v / \partial t$. Hence

$$
\begin{gathered}
\left(\frac{\partial}{\partial t}-\dot{F}^{k l} \nabla_{k} \nabla_{l}\right) v=-\frac{2}{v} \dot{F}^{k l} \nabla_{k} v \nabla_{l} v-\frac{\partial f}{\partial \lambda_{1}}\left(\frac{b^{\prime}}{b}\right)^{\prime}\left(v-v^{-1}\right) \\
-\frac{\partial f}{\partial \lambda_{1}} v\left(v^{-1} \frac{b^{\prime}}{b}-\lambda_{1}\right)^{2}-\frac{\partial f}{\partial \lambda_{2}}\left(\frac{h^{\prime}}{h}\right)^{\prime}\left(v-v^{-1}\right) \\
+f \frac{b^{\prime}}{b}\left(v^{2}-1\right)+\frac{\partial f}{\partial \lambda_{1}} \frac{b^{\prime}}{b}\left(v^{-1} \frac{b^{\prime}}{b}-\lambda_{1}\right)\left(v^{2}-1\right) .
\end{gathered}
$$

Combining $v^{2}$ terms in the second line of (4-6) and applying Euler's identity 
$\frac{\partial f}{\partial \lambda_{1}} \lambda_{1}+\frac{\partial f}{\partial \lambda_{2}} \lambda_{2}=f$ and (2-4), the $v^{2}$ term can be reduced to a linear term:

$\left(f \frac{b^{\prime}}{b}-\frac{\partial f}{\partial \lambda_{1}} \frac{b^{\prime}}{b} \lambda_{1}\right) v^{2}=\left(f \frac{b^{\prime}}{b}-\frac{b^{\prime}}{b}\left(f-\frac{\partial f}{\partial \lambda_{2}} \lambda_{2}\right)\right) v^{2}=\frac{b^{\prime}}{b} \frac{\partial f}{\partial \lambda_{2}} \lambda_{2} v^{2}=\frac{b^{\prime}}{b} \frac{\partial f}{\partial \lambda_{2}} \frac{h^{\prime}}{h} v$.

We then obtain the evolution equation of $v$ as stated in the lemma.

\section{Preserving the property of being a graph and curvature estimates}

In this section, we study HMCF solutions of an axially symmetric torus centered at a closed geodesic satisfying the hypothesis of Theorem 1.1: the initial surface is strictly convex and $\max _{\Sigma_{0}} F<\frac{1}{2}$. Since we will prove many technical estimates, we take this opportunity to outline the overall argument. The main goal of this section is to prove that the evolving surface stays as a graph as it converges to the closed geodesic. As discussed in Section 3, this is equivalent to showing that $v=u^{-1}$ is uniformly bounded for all time (Theorem 5.5). However, we cannot prove the uniform boundedness of $v$ directly using its evolution equation, so the first step is to obtain a weak estimate: $v h \leq C$ where $h(r)=\sinh r$ (Theorem 5.2). This estimate is weaker than $v<C$ since the graph function $r$, thus $\sinh r$, decays to 0 by the barrier argument in Lemma 3.4. We can then deduce by (2-4) that $\lambda_{2}=h^{\prime} / v h=\cosh r /(v \sinh r)$ is uniformly bounded from below. Then, together with Theorem 3.3 we can estimate the ratio of two principal curvatures: $\lambda_{2} / \lambda_{1} \rightarrow \infty$ as $t \rightarrow \infty$ (Corollary 5.3). Equipped with this new estimate for $\lambda_{2} / \lambda_{1}$, we revisit the proof of Theorem 3.3 and obtain the optimal asymptotic upper bound of the HMC (Theorem 5.4): $\lambda_{1} \approx e^{-t}$. Finally, we can prove that the gradient function $v$ is uniformly bounded (Theorem 5.5) and deduce that $\lambda_{2} \approx e^{t}$ thanks to the formula (2-4) for $\lambda_{2}$ available on axially symmetric surfaces. We then conclude in Corollary 5.6 that the principal curvatures of axially symmetric torus behave like those of perfect torus evolving under HMCF as stated in (1-1).

We first consider evolution equations of $\phi(r) v$ where $\phi: \mathbb{R} \rightarrow \mathbb{R}$ is a test function to be chosen later.

Lemma 5.1. The evolution equation for $\phi(r) v$ is given by

$$
\begin{aligned}
& \left(\frac{\partial}{\partial t}-\mathscr{L}\right) \phi v=\phi\left(-\frac{\partial f}{\partial \lambda_{1}}\left(\frac{b^{\prime}}{b}\right)^{\prime}\left(v-\frac{1}{v}\right)-\frac{\partial f}{\partial \lambda_{1}} v\left(\frac{1}{v} \frac{b^{\prime}}{b}-\lambda_{1}\right)^{2}-\frac{\partial f}{\partial \lambda_{2}} \lambda_{2} \frac{b^{\prime}}{b}\right. \\
& \left.+\left[\frac{\partial f}{\partial \lambda_{2}}\left(\frac{h^{\prime}}{h}\right)^{\prime}-\frac{\partial f}{\partial \lambda_{1}}\left(\frac{b^{\prime}}{b}\right)^{2}\right] \frac{1}{v}\right)-f \phi^{\prime}+\left[-\frac{\partial f}{\partial \lambda_{2}}\left(\frac{h^{\prime}}{h}\right)^{\prime}+\frac{\partial f}{\partial \lambda_{2}} \frac{h^{\prime} b^{\prime}}{h b}+\frac{\partial f}{\partial \lambda_{1}}\left(\frac{b^{\prime}}{b}\right)^{2}\right] \phi v \\
& -\frac{\partial f}{\partial \lambda_{1}}\left(-\lambda_{1}+\frac{1}{v} \frac{b^{\prime}}{b}\right) \phi^{\prime}-\frac{\partial f}{\partial \lambda_{2}} \frac{h^{\prime}}{h}\left(v-\frac{1}{v}\right) \phi^{\prime}-\phi^{\prime \prime} \frac{\partial f}{\partial \lambda_{1}}\left(v-\frac{1}{v}\right)-\frac{2}{v} \dot{F}^{k l} \nabla_{k}(\phi v) \nabla_{l} v .
\end{aligned}
$$


Proof. Apply Lemmas 4.2 and 4.3 to

$$
\left(\frac{\partial}{\partial t}-\mathscr{L}\right) \phi v=\phi\left(\frac{\partial}{\partial t}-\mathscr{L}\right) v+v\left(\frac{\partial}{\partial t}-\mathscr{L}\right) \phi-2 \dot{F}^{k l} \nabla_{k} \phi \nabla_{l} v .
$$

Theorem 5.2. We have $h v \leq C$ on $M \times[0, \infty)$, where $h(r)=\sinh r$.

Proof. All the terms in the big parentheses straddling the first and second lines of the equation in Lemma 5.1 are nonpositive, as is the subsequent term $-f \phi^{\prime}$. Substitute $\phi=h$ in that equation. Ignoring all the terms just mentioned since they are nonpositive, we obtain

$$
\begin{aligned}
\left(\frac{\partial}{\partial t}-\mathscr{L}\right) h v \leq[- & \left.\frac{\partial f}{\partial \lambda_{2}}\left(\frac{h^{\prime}}{h}\right)^{\prime}+\frac{\partial f}{\partial \lambda_{2}} \frac{h^{\prime} b^{\prime}}{h b}+\frac{\partial f}{\partial \lambda_{1}}\left(\frac{b^{\prime}}{b}\right)^{2}\right] h v-\frac{\partial f}{\partial \lambda_{1}}\left(\frac{1}{v} \frac{b^{\prime}}{b}-\lambda_{1}\right) h^{\prime} \\
& -\frac{\partial f}{\partial \lambda_{2}} \frac{h^{\prime}}{h}\left(v-\frac{1}{v}\right) h^{\prime}-h^{\prime \prime} \frac{\partial f}{\partial \lambda_{1}}\left(v-\frac{1}{v}\right)-\frac{2}{v} \dot{F}^{k l} \nabla_{k}(h v) \nabla_{l} v \\
= & \left.-\frac{\partial f}{\partial \lambda_{2}}\left(\frac{h^{\prime}}{h}\right)^{\prime}+\frac{\partial f}{\partial \lambda_{2}} \frac{h^{\prime} b^{\prime}}{h b}+\frac{\partial f}{\partial \lambda_{1}}\left(\frac{b^{\prime}}{b}\right)^{2}-\frac{h^{\prime \prime}}{h} \frac{\partial f}{\partial \lambda_{1}}-\left(\frac{h^{\prime}}{h}\right)^{2} \frac{\partial f}{\partial \lambda_{2}}\right] h v \\
& +\left(-h h^{\prime} \frac{\partial f}{\partial \lambda_{1}} \frac{b^{\prime}}{b}+h h^{\prime \prime} \frac{\partial f}{\partial \lambda_{1}}+h^{\prime 2} \frac{\partial f}{\partial \lambda_{2}}\right) \frac{1}{h v} \\
& +h^{\prime} \frac{\partial f}{\partial \lambda_{1}} \lambda_{1}-\frac{2}{v} \dot{F}^{k l} \nabla_{k}(h v) \nabla_{l} v \\
= & -\frac{h v}{\cosh ^{2} r} \frac{\partial f}{\partial \lambda_{1}}+\frac{\cosh ^{2} r}{h v} \frac{\partial f}{\partial \lambda_{2}}+\cosh r \frac{\partial f}{\partial \lambda_{1}} \lambda_{1}-\frac{2}{v} \dot{F}^{k l} \nabla_{k}(h v) \nabla_{l} v .
\end{aligned}
$$

There exist positive constants $C_{0}, C_{1}$, and $C_{2}$ such that

$$
-\frac{1}{\cosh ^{2} r} \frac{\partial f}{\partial \lambda_{1}} \leq-C_{0}, \quad \cosh ^{2} r \frac{\partial f}{\partial \lambda_{2}} \leq C_{1}, \quad \cosh r \frac{\partial f}{\partial \lambda_{1}} \lambda_{1} \leq C_{2},
$$

by Theorem 3.3, Lemma 3.4 and the fact that, if $\lambda_{1}, \lambda_{2}>0$, then

$$
\frac{1}{2} \leq \frac{\partial f}{\partial \lambda_{1}} \leq 1 \quad \text { and } \quad 0 \leq \frac{\partial f}{\partial \lambda_{2}} \leq 1 .
$$

The evolution equation becomes

$$
\left(\frac{\partial}{\partial t}-\mathscr{L}\right) v h \leq-C_{0} v h+C_{1}(v h)^{-1}+C_{2}-\frac{2}{v} \dot{F}^{k l} \nabla_{k}(h v) \nabla_{l} v
$$

and we can apply the maximum principle to obtain a uniform upper bound for $h v$ :

$$
\max _{\Sigma_{t}} h v \leq \max \left\{\frac{1}{2 C_{0}}\left(C_{2}+\sqrt{C_{2}^{2}+4 C_{0} C_{1}}\right), \max _{\Sigma_{0}} h v\right\} .
$$

Corollary 5.3. We have $\lambda_{2}>C_{1}$ and $\lambda_{2} / \lambda_{1} \geq C_{2} e^{t / 2}$ on $\Sigma_{t}$ for all $t \in[0, \infty)$.

Proof. The large principal curvature $\lambda_{2}$ has a uniform lower bound, as can be seen by applying Theorem 5.2 to (2-4). It follows that the ratio $\lambda_{2} / \lambda_{1}$ tends to infinity at the rate $e^{t / 2}$ since $\lambda_{1} \leq C e^{-t / 2}$ from Theorem 3.3. 
We will use the growth estimate of the ratio $\lambda_{2} / \lambda_{1}$ to improve the proof of Theorem 3.3 and squeeze out the optimal upper bound of the harmonic mean curvature $F$. As we shall see below, the ODE associated to the evolution equation of $F$ now has a time dependent coefficient due to the use of growth estimate $\lambda_{2} / \lambda_{1}>C e^{t / 2}$. Therefore, we need to analyze the solution of a nonautonomous ODE in order to establish the optimal upper bound of $F$.

Theorem 5.4. There exist $T>0$ and $C_{1}, C_{2}>0$ such that, for all $t \geq T$,

$$
C_{1} e^{-t} \leq F \leq C_{2} e^{-t} .
$$

Proof. Since Theorem 3.2 provides the lower bound, it is enough to prove the upper bound. We analyze the evolution equation of the harmonic mean curvature $F$ from Theorem 3.3 again:

$$
\begin{aligned}
\left(\frac{\partial}{\partial t}-\mathscr{L}\right) F & =F\left\langle\dot{F}, W^{2}\right\rangle+F\left\langle\dot{F}^{i j}, R_{i 0 j 0}\right\rangle \\
& =\sum_{i} F \frac{\partial f}{\partial \lambda_{i}}\left(\lambda_{i}^{2}+R_{i 0 i 0}\right) \\
& =\sum_{i} F^{3}-\sum_{i} F \frac{\partial f}{\partial \lambda_{i}} \\
& =2 F^{3}-F\left(F^{2} \sum_{i} \lambda_{i}^{-2}\right) \\
& \leq 2 F^{3}-\delta(t) F,
\end{aligned}
$$

where

$$
\delta(t)=\max \left\{\frac{1}{2}, 1-C e^{-t / 2}\right\}
$$

was obtained by observing that $F^{2} \sum_{i=1}^{2} \lambda_{i}^{-2}=\left(\lambda_{1}^{-2}+\lambda_{2}^{-2}\right) /\left(\lambda_{1}^{-1}+\lambda_{2}^{-1}\right)^{2} \geq \frac{1}{2}$ if $\lambda_{i}>0$ and that

$$
F^{2} \sum_{i=1}^{2} \lambda_{i}^{-2} \geq 1-2\left(\frac{\lambda_{2}}{\lambda_{1}}\right)^{-1} \geq 1-C e^{-t / 2}
$$

due to Corollary 5.3. Then, by the maximum principle, $F(x, t) \leq \psi(t)$ for all $(x, t) \in \Sigma \times[0, \infty)$ where $\psi(t)$ is the solution of following nonautonomous ODE:

$$
\frac{d \psi}{d t}=-2 \psi\left(\delta(t) / 2-\psi^{2}\right), \quad \psi(0)=\max _{\Sigma_{0}} F .
$$

Since we are interested in the asymptotic decay rate of the harmonic mean curvature, we will find decay rate of $\psi(t)$ for $t \in[T, \infty)$ for large $T$ by comparing the solution of (5-2) with the solutions of (5-3) and (5-4) below. Note that due to the initial condition $\max _{\Sigma_{0}} F<\frac{1}{2}$ it is not hard to see that $\psi(t) \rightarrow 0$ as $t \rightarrow \infty$; thus we can 
choose large $T$ such that $\psi(T)=\epsilon$ for any given $\epsilon>0$. Consider the ODEs

$$
\begin{aligned}
& \frac{d \bar{\psi}}{d t}=-2 \bar{\psi}\left(\delta / 2-\epsilon^{2}\right), \\
& \frac{d \widehat{\psi}}{d t}=-2 \widehat{\psi}\left(\delta / 2-\bar{\psi}^{2}\right),
\end{aligned}
$$

on the time interval $[T, \infty)$ with conditions $\bar{\psi}(T)=\widehat{\psi}(T)=\epsilon$.

Claim I.

$$
\psi \leq \widehat{\psi} \leq \bar{\psi} \text { for all } t \in[T, \infty) \text {. }
$$

Proof. Since $\psi(T)=\epsilon$ and $\psi$ is nonincreasing for all $t \in[T, \infty)$, from (5-2) and (5-3)

$$
\frac{d}{d t}(\log \psi-\log \bar{\psi})=2\left(\psi^{2}-\epsilon^{2}\right) \leq 0 .
$$

Hence, $\psi \leq \bar{\psi}$ on $[T, \infty)$.

Using this result, we see from (5-2) and (5-4) that

$$
\frac{d}{d t}(\log \psi-\log \widehat{\psi})=2\left(\psi^{2}-\bar{\psi}^{2}\right) \leq 0 .
$$

Hence $\psi \leq \widehat{\psi}$ on $[T, \infty)$. Finally, from (5-3) and (5-4), we have

$$
\frac{d}{d t}(\log \widehat{\psi}-\log \bar{\psi})=2\left(\bar{\psi}^{2}-\epsilon^{2}\right) \leq 0
$$

since $\bar{\psi}(T)=\epsilon$ and $\bar{\psi}$ is nonincreasing. Hence, $\widehat{\psi} \leq \bar{\psi}$ on $[T, \infty)$.

$$
\text { Claim II. } \widehat{\psi}(t) \leq C_{3} e^{-t} \text { for all } t \geq T \text {. }
$$

Proof. Let us find the exact solutions of (5-3) and (5-4). Noting that $\delta(t)=1-C e^{-t / 2}$ for $t \in[T, \infty)$ when $T$ is large, the solution of (5-3) is

$$
\bar{\psi}(t)=\bar{\psi}(T) \exp \left[\left(-1+2 \epsilon^{2}\right) t-2 C e^{-t / 2}+C_{1}\right],
$$

where $C_{1}=\left(1-2 \epsilon^{2}\right) T+2 C e^{-T / 2}$.

Next, substituting (5-5) into (5-4) and integrating in time, we obtain

$$
\begin{aligned}
\log \frac{\widehat{\psi}}{\widehat{\psi}(T)} & =\int_{T}^{t}\left(-1+C e^{-t / 2}+2 \bar{\psi}^{2}\right) d t \\
& =-t-2 C e^{-t / 2}+T+2 C e^{-T / 2}+2 \int_{T}^{t} \bar{\psi}^{2} d t .
\end{aligned}
$$

But

$$
\int_{T}^{t} \bar{\psi}^{2} d t=\bar{\psi}(T)^{2} \int_{T}^{t} \exp \left[2\left(-1+2 \epsilon^{2}\right) t-4 C e^{-t / 2}+2 C_{1}\right] d t \leq C_{2} .
$$

Hence,

$$
\widehat{\psi}(t) \leq C_{3} e^{-t}
$$


Using Claims I and II and the maximum principle, we conclude that

$$
\max _{x \in \Sigma_{t}} F \leq \psi(t) \leq \widehat{\psi}(t) \leq C e^{-t} \text { for all } t \geq T .
$$

We are now in a position to prove that $v$ is uniformly bounded.

Theorem 5.5. There exists a constant $C>0$ such that $v(x, t) \leq C$ for all $(x, t) \in$ $\Sigma \times[0, \infty)$.

Proof. Define a test function $\phi(r)=e^{\mu r^{1+\alpha}}$, where $\mu$ is a positive number to be chosen and $\alpha \in(0,1)$ can be any number. Note that the asymptotic behavior $\phi \rightarrow 1$, $\phi^{\prime} \rightarrow 0$, and $\phi^{\prime \prime} \rightarrow \infty$ as $r \rightarrow 0$ becomes important when it comes to obtaining the desired estimates for the reaction terms in the evolution equation of $\phi(r) v$. In particular,

$$
\phi^{\prime \prime}(r)=\mu(1+\alpha) \alpha r^{-1+\alpha} \phi+\left(\mu(1+\alpha) r^{\alpha}\right)^{2} \phi \geq \mu(1+\alpha) \alpha \max _{\Sigma_{0}} r^{-1+\alpha}
$$

is useful since by choosing $\mu$ large, $\phi^{\prime \prime}$ can be made greater than any large number, but it never becomes infinite in finite time. From Lemma 5.1,

$$
\begin{aligned}
\left(\frac{\partial}{\partial t}-\mathscr{L}\right) \phi v \leq & \left.-\frac{\partial f}{\partial \lambda_{2}}\left(\frac{h^{\prime}}{h}\right)^{\prime}+\frac{\partial f}{\partial \lambda_{2}} \frac{h^{\prime} b^{\prime}}{h b}+\frac{\partial f}{\partial \lambda_{1}}\left(\frac{b^{\prime}}{b}\right)^{2}\right] \phi v \\
& -\frac{\partial f}{\partial \lambda_{1}}\left(v^{-1} \frac{b^{\prime}}{b}-\lambda_{1}\right) \phi^{\prime}-\phi^{\prime \prime} \frac{\partial f}{\partial \lambda_{1}}\left(v-v^{-1}\right)-\frac{2}{v} \dot{F}^{k l} \nabla_{k}(\phi v) \nabla_{l} v \\
\leq & \phi^{\prime \prime}\left(\left[\frac{1}{\phi^{\prime \prime}} \frac{\partial f}{\partial \lambda_{2}} \frac{1}{\sinh ^{2} r}+\frac{1}{\phi^{\prime \prime}} \frac{\partial f}{\partial \lambda_{2}}+\frac{1}{\phi^{\prime \prime}} \frac{\partial f}{\partial \lambda_{1}} \frac{\sinh ^{2} r}{\cosh ^{2} r}-\frac{1}{\phi} \frac{\partial f}{\partial \lambda_{1}}\right] \phi v\right. \\
& \left.+\left[-\frac{\phi^{\prime} \phi}{\phi^{\prime \prime}} \frac{\partial f}{\partial \lambda_{1}} \frac{\sinh r}{\cosh r}+\phi \frac{\partial f}{\partial \lambda_{1}}\right](\phi v)^{-1}+\frac{\phi^{\prime}}{\phi^{\prime \prime}} \frac{\partial f}{\partial \lambda_{1}} \lambda_{1}\right) \\
& -\frac{2}{v} \dot{F}^{k l} \nabla_{k}(\phi v) \nabla_{l} v .
\end{aligned}
$$

Let us first examine the coefficient of $\phi v$, in the third line of (5-7). Since $F \approx e^{-t}$ by Theorem 5.4, $\sinh r \approx e^{-t}$ by Lemma 3.4, and $\lambda_{2}>C$ by Corollary 5.3, the first term is

$$
\frac{\partial f}{\partial \lambda_{2}} \frac{1}{\sinh ^{2} r}=\frac{f^{2} \lambda_{2}^{-2}}{\sinh ^{2} r} \leq C .
$$

By Lemma 3.4, (5-1), (5-6), and (5-8), we see that the first three terms can be made arbitrarily small if we choose a large $\mu$. On the other hand, the last term in the third line of (5-7) is strictly negative since we can find a constant $C_{0}>0$ such that $\phi^{-1} \partial f / \partial \lambda_{1}>C_{0}$; thus there is a constant $C_{1}>0$ such that

$$
\frac{1}{\phi^{\prime \prime}} \frac{\partial F}{\partial \lambda_{2}} \frac{1}{\sinh ^{2} r}+\frac{1}{\phi^{\prime \prime}} \frac{\partial F}{\partial \lambda_{2}}+\frac{1}{\phi^{\prime \prime}} \frac{\partial F}{\partial \lambda_{1}}\left(\frac{\sinh r}{\cosh r}\right)^{2}-\frac{1}{\phi} \frac{\partial f}{\partial \lambda_{1}} \leq-C_{1} .
$$


Using similar argument, we see that the rest of the terms in the fourth line of (5-7) can be uniformly bounded above, so the evolution equation becomes

$$
\left(\frac{\partial}{\partial t}-\mathscr{L}\right) \phi v \leq \phi^{\prime \prime}\left(-C_{1} \cdot \phi v+C_{2}(\phi v)^{-1}+C_{3}\right)-\frac{2}{v} \dot{F}^{k l} \nabla_{k}(\phi v) \nabla_{l} v,
$$

and we can apply the maximum principle to conclude that on $\Sigma \times[0, \infty)$,

$$
v \leq \phi v \leq \max \left\{\max _{\Sigma_{0}} \phi v, \frac{C_{3}+\sqrt{C_{3}^{2}+4 C_{1} C_{2}}}{2 C_{1}}\right\} .
$$

Due to the formula (2-4) for $\lambda_{2}$ available on axially symmetric surfaces, the uniform boundedness of $v$ implies that $\lambda_{2} \approx 1 / \sinh r \approx e^{t}$. Together with the asymptotic estimate for $\lambda_{1}$ from Theorem 5.4, we have shown that the principal curvatures of an axially symmetric torus evolving by HMCF have the same asymptotic curvature estimates as the perfect torus shrinking under HMCF as stated in (1-1).

Corollary 5.6. $\lambda_{1} \approx e^{-t}, \lambda_{2} \approx e^{t}$, and $\lambda_{1} \lambda_{2} \approx 1$ on $\Sigma \times[0, \infty)$.

Note that uniform boundedness of $v$ implies that $\left|r_{z}\right|$ is uniformly bounded. In fact, more can be said about $\left|r_{z}\right|$ and $\left|r_{z z}\right|$ if we apply the results of Theorems 5.4 and 5.5 to the formula (2-3) for $\lambda_{1}$. Moreover, we can deduce a better estimate for $\lambda_{2}$.

Corollary 5.7. We have $\max _{z \in S^{1}}\left|r_{z z}\right| \leq C_{1} e^{-t}, \max _{z \in S^{1}}\left|r_{z}\right| \leq C_{2} e^{-t}$, and

$$
\max _{z \in S^{1}}|v-1| \rightarrow 0, \quad \frac{\lambda_{2}}{\operatorname{coth} r} \rightarrow 1 \quad \text { as } t \rightarrow \infty .
$$

Proof. Solving for $r_{z z}$ in (2-3), we obtain

$$
r_{z z}=\frac{1}{b}\left[-\lambda_{1}\left(r_{z}^{2}+b^{2}\right)^{3 / 2}+\left(2 r_{z}^{2}+b^{2}\right) b^{\prime}\right] .
$$

Using that $\left|r_{z}\right|$ is uniformly bounded and both $\lambda_{1}$ and $b^{\prime}=\sinh r$ decrease at the rate $e^{-t}$,

$$
\left|r_{z z}\right| \leq \frac{\left(r_{z}^{2}+b^{2}\right)^{3 / 2}}{b} \lambda_{1}+\frac{2 r_{z}^{2}+b^{2}}{b} b^{\prime} \leq C e^{-t} .
$$

Since $r$ is a function defined on $S^{1}$, the derivative $r_{z}$ cannot have a sign; that is, at each time $t$, there is $z_{0}(t)$ such that $r_{z}\left(z_{0}(t), t\right)=0$. Then,

$$
\max _{z \in S^{1}}\left|r_{z}(z, t)\right|=\max _{S^{1}}\left|r_{z}(z, t)-r_{z}\left(z_{0}(t), t\right)\right| \leq \max _{S^{1}} \int_{z_{0}(t)}^{z}\left|r_{z z}(s, t)\right| d s \leq C e^{-t} .
$$

Now, by the definition of $v$ we see that $v \rightarrow 1$ uniformly in space and time, and from the formula (2-4) for $\lambda_{2}$ we obtain uniform convergence $\lambda_{2} \rightarrow \operatorname{coth} r$.

\section{Acknowledgements}

I would like to express my gratitude to my advisor Robert Gulliver for his continuous support and encouragement throughout my Ph.D. studies. 


\section{References}

[Andrews 1994a] B. Andrews, "Contraction of convex hypersurfaces in Euclidean space", Calc. Var. Partial Differential Equations 2:2 (1994), 151-171. MR 97b:53012 Zbl 0805.35048

[Andrews 1994b] B. Andrews, "Contraction of convex hypersurfaces in Riemannian spaces", $J$. Differential Geom. 39:2 (1994), 407-431. MR 95b:53044 Zbl 0797.53044

[Andrews et al. 2013] B. Andrews, J. McCoy, and Y. Zheng, "Contracting convex hypersurfaces by curvature", Calc. Var. Partial Differential Equations 47:3-4 (2013), 611-665. MR 3070558 Zbl 06187283

[Cabezas-Rivas and Miquel 2009] E. Cabezas-Rivas and V. Miquel, "Volume-preserving mean curvature flow of revolution hypersurfaces in a rotationally symmetric space", Math. Z. 261:3 (2009), 489-510. MR 2009m:53175 Zbl 1161.53053

[Caputo and Daskalopoulos 2009] M. C. Caputo and P. Daskalopoulos, "Highly degenerate harmonic mean curvature flow", Calc. Var. Partial Differential Equations 35:3 (2009), 365-384. MR 2010f:35218 Zbl 1179.35174

[Daskalopoulos and Hamilton 1999] P. Daskalopoulos and R. Hamilton, "The free boundary in the Gauss curvature flow with flat sides”, J. Reine Angew. Math. 510 (1999), 187-227. MR 2000g:53081 Zbl 0931.53031

[Daskalopoulos and Hamilton 2006] P. Daskalopoulos and R. Hamilton, "Harmonic mean curvature flow on surfaces of negative Gaussian curvature", Comm. Anal. Geom. 14:5 (2006), 907-943. MR 2007i:53072 Zbl 1127.53058

[Daskalopoulos and Sesum 2010] P. Daskalopoulos and N. Sesum, "The harmonic mean curvature flow of nonconvex surfaces in $\mathbb{R}^{3}$ ", Calc. Var. Partial Differential Equations 37:1-2 (2010), 187-215. MR 2010k:53096 Zbl 1189.53064

[Dieter 2005] S. Dieter, "Nonlinear degenerate curvature flows for weakly convex hypersurfaces", Calc. Var. Partial Differential Equations 22:2 (2005), 229-251. MR 2005h:53117 Zbl 1076.53079

[Gulliver and $\mathrm{Xu}$ 2009] R. Gulliver and G. Xu, "Examples of hypersurfaces flowing by curvature in a Riemannian manifold", Comm. Anal. Geom. 17:4 (2009), 701-719. MR 2011e:53102 Zbl 1197.53084

[Huisken 1984] G. Huisken, "Flow by mean curvature of convex surfaces into spheres", J. Differential Geom. 20:1 (1984), 237-266. MR 86j:53097 Zbl 0556.53001

Received May 23, 2013.

\section{CHRISTOPHER KIM}

SCHOOL OF MATHEMATICS

UNIVERSITY OF MINNESOTA

127 VINCENT HALL

206 CHURCH ST. SE

MINNEAPOLIS, MN 55455

UNITED STATES

cmkim@umn.edu 


\title{
PACIFIC JOURNAL OF MATHEMATICS
}

\author{
msp.org/pjm
}

Founded in 1951 by E. F. Beckenbach (1906-1982) and F. Wolf (1904-1989)

\section{EDITORS}

V. S. Varadarajan (Managing Editor)

Department of Mathematics

University of California

Los Angeles, CA 90095-1555

pacific@math.ucla.edu

Paul Balmer

Department of Mathematics

University of California

Los Angeles, CA 90095-1555

balmer@math.ucla.edu

Daryl Cooper

Department of Mathematics

University of California

Santa Barbara, CA 93106-3080 cooper@math.ucsb.edu

Jiang-Hua $\mathrm{Lu}$

Department of Mathematics

Pokfulam Rd., Hong Kong jhlu@maths.hku.hk
The University of Hong Kong

Don Blasius

Department of Mathematics University of California

Los Angeles, CA 90095-1555

blasius@math.ucla.edu

Robert Finn

Department of Mathematics Stanford University

Stanford, CA 94305-2125

finn@math.stanford.edu

Sorin Popa

Department of Mathematics

University of California

Los Angeles, CA 90095-1555 popa@math.ucla.edu

Paul Yang

Department of Mathematics Princeton University

Princeton NJ 08544-1000

yang@math.princeton.edu

\section{PRODUCTION}

Silvio Levy, Scientific Editor, production@msp.org

\section{SUPPORTING INSTITUTIONS}

ACADEMIA SINICA, TAIPEI

CALIFORNIA INST. OF TECHNOLOGY

INST. DE MATEMÁTICA PURA E APLICADA

KEIO UNIVERSITY

MATH. SCIENCES RESEARCH INSTITUTE

NEW MEXICO STATE UNIV.

OREGON STATE UNIV.

\author{
STANFORD UNIVERSITY \\ UNIV. OF BRITISH COLUMBIA \\ UNIV. OF CALIFORNIA, BERKELEY \\ UNIV. OF CALIFORNIA, DAVIS \\ UNIV. OF CALIFORNIA, LOS ANGELES \\ UNIV. OF CALIFORNIA, RIVERSIDE \\ UNIV. OF CALIFORNIA, SAN DIEGO \\ UNIV. OF CALIF., SANTA BARBARA
}

\author{
Vyjayanthi Chari \\ Department of Mathematics \\ University of California \\ Riverside, CA 92521-0135 \\ chari@math.ucr.edu \\ Kefeng Liu \\ Department of Mathematics \\ University of California \\ Los Angeles, CA 90095-1555 \\ liu@math.ucla.edu \\ Jie Qing \\ Department of Mathematics \\ University of California \\ Santa Cruz, CA 95064 \\ qing@cats.ucsc.edu
}

These supporting institutions contribute to the cost of publication of this Journal, but they are not owners or publishers and have no responsibility for its contents or policies.

See inside back cover or msp.org/pjm for submission instructions.

The subscription price for 2014 is US \$410/year for the electronic version, and \$535/year for print and electronic.

Subscriptions, requests for back issues and changes of subscribers address should be sent to Pacific Journal of Mathematics, P.O. Box 4163, Berkeley, CA 94704-0163, U.S.A. The Pacific Journal of Mathematics is indexed by Mathematical Reviews, Zentralblatt MATH, PASCAL CNRS Index, Referativnyi Zhurnal, Current Mathematical Publications and Web of Knowledge (Science Citation Index).

The Pacific Journal of Mathematics (ISSN 0030-8730) at the University of California, c/o Department of Mathematics, 798 Evans Hall \#3840, Berkeley, CA 94720-3840, is published twelve times a year. Periodical rate postage paid at Berkeley, CA 94704, and additional mailing offices. POSTMASTER: send address changes to Pacific Journal of Mathematics, P.O. Box 4163, Berkeley, CA 94704-0163.

PJM peer review and production are managed by EditFLOW ${ }^{\circledR}$ from Mathematical Sciences Publishers.

\section{PUBLISHED BY}

mathematical sciences publishers

nonprofit scientific publishing

http://msp.org/

(C) 2014 Mathematical Sciences Publishers 


\section{PACIFIC JOURNAL OF MATHEMATICS}

Volume $268 \quad$ No. $1 \quad$ March 2014

AlEXANDRE PAIVA BARRETO

A transport inequality on the sphere obtained by mass transport

DARIO CORDERO-ERAUSQUIN

A cohomological injectivity result for the residual automorphic spectrum of $\mathrm{GL}_{n}$

HARALD GROBNER

Gradient estimates and entropy formulae of porous medium and fast diffusion equations for the Witten Laplacian

GUANGYUE HUANG and HAIZHONG LI

Controlled connectivity for semidirect products acting on locally finite trees

KeITH JoNES

An indispensable classification of monomial curves in $\mathbb{A}^{4}(\mathbb{k})$

ANARGYROS KATSABEKIS and IGNACIO OJEDA

Contracting an axially symmetric torus by its harmonic mean curvature

CHRISTOPHER KIM

Composition operators on strictly pseudoconvex domains with smooth symbol

HYUNGWOON KOO and SONG-YING LI

The Alexandrov problem in a quotient space of $\mathbb{U}^{2} \times \mathbb{R}$

AnA Menezes

Twisted quantum Drinfeld Hecke algebras

DEEPAK NAIDU

$L^{p}$ harmonic 1-forms and first eigenvalue of a stable minimal hypersurface

KEOMKYO SEO

Reconstruction from Koszul homology and applications to module and derived categories

RYO TAKAHASHI

A virtual Kawasaki-Riemann-Roch formula 